\title{
How to Find a Publisher in Political Science
}

\author{
Christopher J. Kelaher, Chatham House Publishers \\ Edward Artinian, Chatham House Publishers
}

The questions asked most frequently of editors in attendance at APSA meetings are "How do I get my book published?" and "Who should publish my book?" To help prospective authors, we have compiled a list of companies and editors working in political science. We have also tried to identify important questions to ask when seeking publication. This information should provide a valuable base from which to work when searching for a publisher. We hope it will save authors and publishers time, money, and effort.

The criteria for inclusion in our list are attendance and exhibits at national and regional political science meetings and advertising in publications such as the American Political Science Review. We should point out that new publishers are always entering the political science field.

Marketing strategies differ from one organization to another. Thus, this list may not be inclusive; we apologize for any omissions and promise to amend future lists in accordance with additional information we may receive.

It is possible for authors to pose a number of questions requiring quantitative responses from publishers. The answers to such questions reveal a great deal about a publisher's expectations for a book, its market, and the publisher's approach to that market. These questions might include:

(1) How many copies would be printed, in cloth and/or simultaneously in paper?

(2) At what price would the book be sold?

(3) How many free copies, if any, does the publisher expect to give away? (Adoption-market vehicles will demand more free copies.)

It is advisable for authors to examine a publisher's catalog, exhibits, and advertising. These also reveal much about the company's products and approach, and they allow a writer to gain a feel for that company's presence in the marketplace.

\section{Political Science Editors}

Basic Books

Martin Kessler

10 E. $53 \mathrm{~d}$ Street

New York, NY 10022

Phone: (212) 207-7072

Fax: (212) 207-7203

Basil Blackwell

John Davey

3 Cambridge Center

Cambridge, MA 02142

Phone: (617) 225-0430

Fax: (617) 621-9582

Brassey's (US), Inc.

Franklin D. Margiotta

8000 Westpark Drive, 1st Floor

McLean, VA 22102

Phone: (703) 442-4535

Fax: (703) 790-9063

Brookings Institution

Nancy Davidson

1775 Massachusetts Avenue, NW

Washington, DC 20036

Phone: (202) 797-6260

Fax: (202) 797-6004

Brooks/Cole Publishing Co.

Cynthia Stormer

511 Forest Lodge Road

Pacific Grove, CA 93950

Phone: (408) 373-0728

Fax: (408) 375-6414

Brown and Benchmark

Ed Laube

25 Kessel Court

Madison, WI 53711

Phone: (608) 273-0040

Fax: (608) 277-7351

University of California Press

Naomi Schneider

2120 Berkeley Way

Berkeley, CA 94720

Phone: (415) 642-6715

Fax: (415) 643-7127

Cambridge University Press

Emily Loose

$40 \mathrm{~W}$. 20th Street

New York, NY 10011

Phone: (212) 924-3900

Fax: (212) 691-3239
Chatham House Publishers

Edward Artinian/Christopher Kelaher

Box One

Chatham, NJ 07928

Phone: (201) 635-2059

Fax: (201) 635-9366

University of Chicago Press

John Tryneski

5801 Ellis Avenue

Chicago, IL 60637

Phone: (312) 702-7648

Fax: (312) 702-9756

Columbia University Press

Kate Wittenberg

562 W. 113th Street

New York, NY 10025

Phone: (212) 316-7100

Fax: (212) 316-7169

Cornell University Press

Roger Haydon (US politics)/Peter Agree

Box 250, 24 Roberts Place

Ithaca, NY 14851

Phone: (607) 257-7000

Fax: (607) 257-3552

CQ Press

Brenda Carter, Jeanne Ferris, David Tarr

1414 22d Street, NW

Washington, DC 20037

Phone: (202) 887-8641, (202) 887-8642 (202) $887-8640$

Fax: (202) 728-1863

Crane Russak/Taylor \& Francis

Todd Baldwin

1101 Vermont Avenue, NW, Suite 200

Washington, DC 20005

Phone: (202) 289-2174

Fax: (202) 289-3665

Duke University Press

Lawrence J. Malley

Box 6697, College Station

Durham, NC 27708

Phone: (919) 684-2173

Fax: (919) 684-8644

Dushkin Publishing Group

Irving Rockwood

Sluice Dock

Guilford, CT 06437

Phone: (203) 453-4351

Fax: (203) 453-6000

Free Press

Peter Dougherty, Adam Bellow

866 Third Avenue

New York, NY 10022

Phone: (212) 702-4214, (212) 702-6806

Fax: (212) 605-9364 
Greenwood Publishing Group

Mildred Vasan

Box 5007, 88 Post Road W

Westport, CT 06881

Phone: (203) 226-3571

Fax: (203) 222-1502

Harcourt Brace Jovanovich

David Tatom

301 Commerce Street, Suite 3700

Ft. Worth, TX 76102

Phone: (817) 334-7529

Fax: (817) 334-0947

HarperCollins Publishers

Lauren Silverman, Maria Hartwell

10 E. 53d Street

New York, NY 10022

Phone: (212) 207-7299, (212) 207-7855

Fax: (212) 207-7134

Harvard University Press

Aida Donald

79 Garden Street

Cambridge, MA 02138

Phone: (617) 495-4703

Fax: (617) 496-4677

D.C. Heath

Paul Smith

125 Spring Street

Lexington, MA 02173

Phone: (617) 860-1136

Fax: (617) 860-1587

Holmes \& Meier Publishers

Katharine Turok

30 Irving Place

New York, NY 10003

Phone: (212) 254-4100

Fax: (212) 254-4104

Houghton Mifflin Company

Margaret Seawell

One Beacon Street

Boston, MA 02108

Phone: (617) 725-5858

Fax: (617) 573-4997

Humanities Press International

Keith Ashfield

165 First Avenue

Atlantic Highlands, NJ 07716

Phone: (908) 872-1441

Fax: (908) 872-0717

University of Illinois Press

Richard Martin

P.O. Box 4348

Chicago, IL 60614

Phone: (312) 996-5080

Indiana University Press

Robert Sloan

601 N. Morton Street

Bloomington, IN 47405

Phone: (812) 855-7561

Fax: (812) 855-7931

Johns Hopkins University Press

Henry Tom

701 W. 40th, Suite 275

Baltimore, MD 21211

Phone: (410) 516-6908
Fax: (410) 516-6998

Jossey-Bass Publishers

Alan R. Shrader

350 Sansome Street

San Francisco, CA 94104

Phone: (415) 433-1740

Fax: (415) 433-0499

University Press of Kansas

Michael Briggs/Fred Woodward

2501 West 15th Street

Lawrence, KS 66049

Phone: (913) 864-4154

Fax: (913) 864-4586

Kendall/Hunt Publishing

Tom Gantz

2460 Kerper Blvd.

Dubuque, IA 52004

Phone: (319) 588-1451

Fax: (319) 589-4666

University Press of Kentucky

Wm. Jerome Crouch/Kevin Murphy

663 S. Limestone Street

Lexington, KY 40508

Phone: (606) 257-2951

Fax: (606) 257-2984

Longman Publishing Group

David Estrin

95 Church Street

White Plains, NY 10601

Phone: (914) 993-5000

Fax: (914) 997-8115

Louisiana State University Press

Margaret Dalrymple

Baton Rouge, LA 70893

Phone: (504) 388-6294

Fax: (504) 388-6461

Lynne Rienner Publishers

Lynne Rienner/Martha Peacock

1800 30th St., Suite 314

Boulder, CO 80301

Phone: (303) 444-6684

Fax: (303) 444-0824

McGraw-Hill

Peter Labella

575 Boylston Street

Boston, MA 02116

Phone: (617) 262-1160

Fax: (617) 437-0395

Macmillan Publishing company

Bruce Nichols

866 Third Avenue

New York, NY 10022

Phone: (212) 702-6839

Fax: (212) 605-9372

University of Michigan Press

Colin Day

Box 1104, 839 Greene Street

Ann Arbor, MI 48106

Phone: (313) 764-4388

Fax: (313) 936-0456

University of Minnesota Press

Lisa Freeman

2037 University Ave., SE
Minneapolis, MN 55414

Phone: (612) 624-2516

Fax: (612) 626-7313

Nelson-Hall Publishers

Richard O. Meade

111 N. Canal Street

Chicago, IL 60606

Phone: (312) 930-9446

Fax: (312) 930-5903

State University of New York Press

Clay Morgan

State University Plaza

Albany, NY 12246

Phone: (518) 472-5042

Fax: (518) 472-5038

University of North Carolina Press

Kate Torrey

Box 2288

Chapel Hill, NC 27515

Phone: (919) 966-3561

Fax: (919) 966-3829

W.W. Norton and Co.

Don Fusting/Roby Harrington

500 Fifth Avenue

New York, NY 10110

Phone: (212) 354-5500

Fax: (212) 869-0856

Oxford University Press

David Roll

200 Madison Avenue

New York, NY 10016

Phone: (212) 679-7300, ext. 7217

Fax: (212) 725-2972

F.E. Peacock Publishers

Leo A. W. Wiegman

P.O. Box 397

Itasca, IL 60143

Phone: (708) 350-0777

Fax: (708) 350-0588

Pennsylvania State University Press Sanford Thatcher/Peter Potter 820 N. University Drive, Suite C University Park, PA 16802

Phone: (814) 865-1327

Fax: (814) 863-1408

University of Pittsburgh Press

Frank Moone

127 N. Bellefield Avenue

Pittsburgh, PA 15260

Phone: (412) 624-4110

Fax: (412) 624-7380

Praeger Publishers

James Dunton

One Madison Avenue

New York, NY 10010

Phone: (212) 685-7647

Fax: (212) 685-0285

Prentice Hall

Julia Berrisford

Route 9W

Englewood Cliffs, NJ 07632

Phone: (201) 592-2251

Fax: (201) 592-2275 
Princeton University Press

Malcolm DeBevoise

41 William Street

Princeton, NJ 08540

Phone: (609) 258-4922

Fax: (609) 258-6305

Routledge

Cecelia Cancellaro

29 W. 35th Street

New York, NY 10001

Phone: (212) 244-3336, ext. 342

Fax: (212) 563-2269

Rowman \& Littlefield

Jonathan Sisk

4720 Boston Way

Lanham, MD 20706

Phone: (301) 306-0400

Fax: (301) 459-2118

Sage Publications

Mitch Allen

1630 N. Main Street, Suite 367

Walnut Creek, CA 94596

Phone: (510) 938-7243

Fax: (510) 933-9720

St. Martin's Press

Don Reisman (college)

Simon Winder (scholarly)

175 Fifth Avenue

New York, NY 10010

Phone: (212) 674-5151

Fax: (212) 420-9314

M.E. Sharpe

Michael Weber

80 Business Park Drive

Armonk, NY 10504

Phone: (914) 273-1800

Fax: (914) 273-2106

University of South Carolina Press

David Caffry
1716 College Street

Columbia, SC 29208

Phone: (803) 777-4859

Fax: (803) 777-0160

Southern Illinois University Press

Richard DeBacher

Box 3697

Carbondale, IL 62901

Phone: (618) 453-6625

Fax: (618) 453-1221

Stanford University Press

Muriel Bell

Stanford, CA 94305

Phone: (415) 725-0824

Fax: (415) 725-3457

Temple University Press

Michael Ames

1601 N. Broad Street, USB 306

Philadelphia, PA 19122

Phone: (215) 787-8787

Fax: (215) 787-4719

University of Texas Press

Theresa May

Box 7819

Austin, TX 78713

Phone: (512) 320-0668

Fax: (512) 471-4278

Transaction Publishers

Irving Louis Horowitz

Rutgers University, Bldg. \#4051

New Brunswick, NJ 08903

Phone: (908) 932-2280

Fax: (908) 932-3138

University Press of America

Maureen Muncaster

4720 Boston Way

Lanham, MD 20706

Phone: (301) 306-0400

Fax: (302) 459-2118
West Publishing Co.

Clyde Perlee

P.O. Box 5090

Thousand Oaks, CA 91359

Phone: (818) 707-0026

Fax: (818) 707-7016

Westview Press

Jennifer Knerr

5500 Central Avenue

Boulder, CO 80301

Phone: (303) 444-3541

Fax: (303) 449-3356

University of Wisconsin Press

Barbara Hanrahan

114 N. Murray Street

Madison, WI 53715

Phone: (608) 262-4928

Fax: (608) 262-7560

Yale University Press

John Covell

302 Temple Street

New Haven, CT 06511

Phone: (203) 432-0902

Fax: (203) 432-0948

\section{About the Authors}

Christopher J. Kelaher did his undergraduate work at the University of Dayton and received a master's degree in political science from the University of Wisconsin. Edward Artinian has been a member of APSA since 1961; he did his undergraduate work at Brown University and received a master's degree in business from Columbia University.

\title{
An Epidemic of Cheating?*
}

\author{
Donald Chisholm, University of California, Los Angeles
}

An essay final exam is given, the exams graded and returned to the students. One, dissatisfied with her grade, steals her roommate's exam, puts the cover from her bluebook on it, and tells the instructor that there must have been an error in the way her grade was recorded.

A student copies an entire article from the American Political Science Review and turns it in as his own. The instructor is initially impressed with the paper, but becomes sus- picious when the student discusses how he computed the tau-beta's.

Each member of a group of five students in one course prepares for a single section of the syllabus, and then trades answers with other members of the group during the final exam.

These tales are true. They happen to come from my experiences at several universities. Unfortunately, these are not rare, isolated occurrences. To judge from reports in the popular press, we are mired in an ever deepening morass of academic dishonesty. "An Epidemic of College Cheating!" declares one recent article in the Los Angeles Times. ${ }^{1}$ A second trumpets: "Study Finds Cheating Joins 3 Rs as a Basic College Skill." 2 The Chronicle of Higher Education reports "Survey at Rutgers Suggests that Cheating May Be on the Rise at Large Universities." ${ }^{3}$ A Maryland university official tells us that "Honesty is becoming a more negoti- 